\title{
Permutations sortable by two stacks in parallel and quarter plane walks
}

\author{
Michael Albert $\|^{1 / k}$ and Mireille Bousquet-Mélou虾 \\ ${ }^{1}$ Department of Computer Science, University of Otago, New Zealand \\ ${ }^{2}$ CNRS, LaBRI, Université de Bordeaux, France
}

\begin{abstract}
At the end of the 1960s, Knuth characterised in terms of forbidden patterns the permutations that can be sorted using a stack. He also showed that they are in bijection with Dyck paths and thus counted by the Catalan numbers. Subsequently, Pratt and Tarjan asked about permutations that can be sorted using two stacks in parallel. This question is significantly harder, and the associated counting question has remained open for 40 years. We solve it by giving a pair of equations that characterise the generating function of such permutations.

The first component of this system describes the generating function $Q(a, u)$ of square lattice loops confined to the positive quadrant, counted by the length and the number of North-West and East-South factors. Our analysis of the asymptotic number of sortable permutations relies at the moment on two intriguing conjectures dealing with this series. Given the recent activity on walks confined to cones, we believe them to be attractive per se. We prove these conjectures for closed walks confined to the upper half plane, or not confined at all.

Résumé. Nous énumérons les permutations triables par deux piles en parallèle. Cette question était restée ouverte depuis les travaux de Knuth, Pratt et Tarjan dans les années 70. Notre solution consiste en une paire d'équations qui caractérisent la série génératrice. La première composante de ce système décrit la série $Q(a, u)$ des chemins fermés confinés dans le quart de plan positif, comptés selon leur longueur et le nombre de facteurs Nord-Ouest ou Est-Sud. Notre analyse du comportement asymptotique du nombre de permutations triables repose à ce stade sur deux conjectures remarquables portant sur $Q(a, u)$. Nous les prouvons pour les chemins fermés non confinés, ou confinés au demi-plan supérieur.
\end{abstract}

Keywords: permutations, stacks, quarter plane walks, generating functions

\section{Introduction}

If we have a device whose only ability is to rearrange certain sequences of objects, it is very natural to ask "What rearrangements can my device produce?" When the device is an abstract one that can operate on sequences of any size, this becomes a combinatorial question. Such questions were apparently first considered by Knuth who dealt with the case where the device was a stack, i.e. a storage mechanism operating in a last in, first out manner (Figure 1).

*Email: michael.albertecs.otago.ac.nz

†Email: mireille.bousquetelabri.fr

1365-8050 @ 2014 Discrete Mathematics and Theoretical Computer Science (DMTCS), Nancy, France 

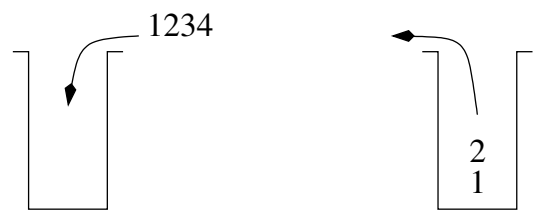

34

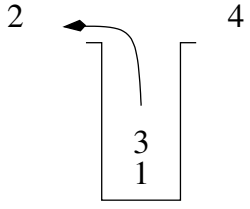

23

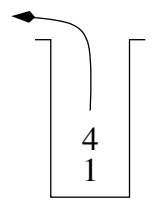

Fig. 1: Four steps in the sequence of operations that outputs 2341 from 1234 using a single stack. Each arrow shows an operation that is about to be performed.

Using a stack it is clear that the input sequence $a b c$ cannot be transformed into the output sequence $c a b$ as, in order for $c$ to be the first element output, both $a$ and $b$ must be in the stack together but then they will be output as $b a$ and not as $a b$. For a single stack this is the only restriction. In modern language, if the input is taken to be $12 \cdots n$, then the achievable permutations are those that avoid the pattern 312 . Moreover, as the stack operations that will produce an output sequence from a given input sequence are easily seen to be uniquely determined, it is also routine to count such permutations, which turn out to be enumerated by the Catalan numbers. This is described in Section 2.2.1 of The Art of Computer Programming [13]. Knuth also establishes there similar results for input-restricted deques (double-ended queues).

Knuth's investigations, nicely described in terms of "railway yard switching networks", were extended by Even \& Itai [9], Pratt [18] and Tarjan [20] who considered more general networks of stacks and queues, including a small network consisting of two stacks in parallel (Figure 2). This work was foundational for the study of permutation classes which can loosely be described as those collections of permutations that are closed by taking sub-permutations, and normalizing them. In our case, we observe indeed that any sub-permutation of a permutation that can be produced using two parallel stacks can itself be produced by this device. The study of permutation classes has been an active and growing field, often concentrating on enumeration, but also dealing with structural properties of these classes. For some general discussions and background see the books [3, 12, 15], and [2] for a survey on stack-sorting.
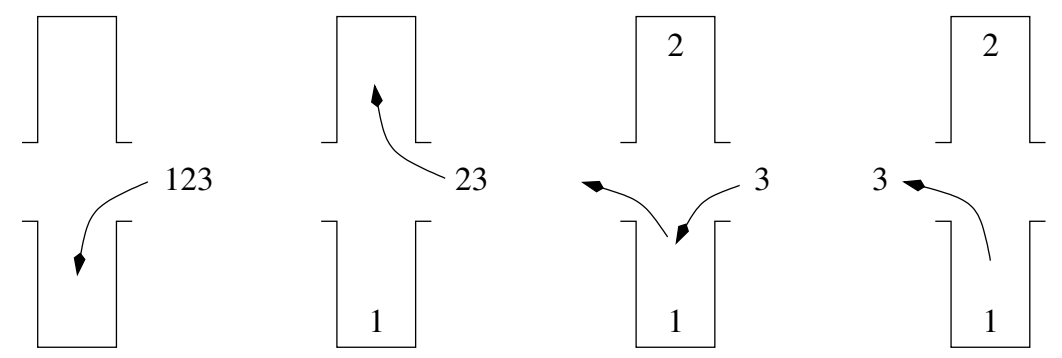

Fig. 2: The permutation 312 cannot be produced with a single stack, but can be produced with two parallel stacks as shown here. Note that several distinct sequences of operations produce it.

Despite this activity, most problems related to the rearranging power of Knuth's railway's switching networks have turned out to be very hard. For networks consisting of two stacks, the case of parallel stacks seems a bit more manageable than that of two stacks in series. For instance, the list of minimal permutations that cannot be achieved by two parallel stacks has been known since 1973 [18], but for two 
stacks in series it is only known to be infinite [16]. Similarly, it has just been proved this year that one can decide in polynomial time if a permutation can be sorted by two stacks in series [17], while the corresponding result was proved in 1992 for two parallel stacks [9, 22]. However, the natural questions "How many permutations of length $n$ can be generated by two stacks in series, or by two stacks in parallel?" have remained equally open for forty years.

We answer the latter question in this paper, by giving a system of two functional equations that defines the generating function $\sum_{n} s_{n} t^{n}$, where $s_{n}$ is the number of permutations of length $n$ that can be produced with two parallel stacks. We also determine the exponential growth of the numbers $s_{n}$, modulo some conjectures that deal with square lattice walks confined to the quarter plane. These walks naturally encode the admissible sequences of stack operations, in the same way as Dyck paths do in the case of a single stack. Our conjectures deal with the enumeration of quarter plane walks counted by the length and by the number of corners of certain types. Walks confined to a quadrant have attracted a lot of attention in the past decade (see e.g. [4, 5, 6, 7, 14, 11]), and we believe that our conjectures are of interest quite independently of the original stack sorting question.

Finally we remark that in this metaphor of "devices rearranging input" there are two common viewpoints. As described above, Knuth and Pratt tended to view the input as arriving in fixed order $12 \cdots n$ and then the question is "How many permutations can be generated?". Tarjan on the other hand tended to think of the objective being to sort the input permutation, so the enumerative question becomes "How many permutations can be sorted?". Of course, passing to inverses, the two viewpoints are equivalent to one another. We will be adopting the first one.

The outline of this extended abstract is as follows. In Section 2, we describe a set of canonical operation sequences such that each permutation that can be produced using two parallel stacks is obtained by exactly one canonical operation sequence. In Section 3 , we establish a system of functional equations that characterises the generating function of canonical sequences, and thus, of permutations that can be produced by two parallel stacks. The first equation in this system defines the generating function of quarter plane walks, weighted by their length and the number of North-West and East-South factors (also called corners). In Section 4, we state two conjectures about this generating function, and provide evidence for them by demonstrating that they hold if we only impose on walks a half plane restriction, or no restriction at all. In Section 5 we derive from our system of equations the exponential growth of the number of permutations of length $n$ produced by two parallel stacks, assuming the conjectures of Section 4 Proofs and more detailed discussions of a number of issues are omitted, but are included in the full version of this paper, available from the arXiv

\section{Canonical operation sequences}

Throughout this paper we consider the action of two stacks in parallel, and attempt to count permutations of length $n$ that such a machine can generate. The primary issue in this question, as opposed to the case of a single stack considered by Knuth [13], is that there is no one-to-one correspondence between sequences of operations of the machine, and the permutations they produce. That is, several sequences of operations may produce the same permutation: we then say that they are equivalent. The most obvious case is that of the identity permutation of length $n$ : there are at least $2^{n}$ ways to produce it using two stacks (alternate input and output operations, allowing the freedom of choice as to which stack to use).

In this section we define a family of operation sequences, called canonical, such that each operation sequence is equivalent to exactly one canonical sequence. Canonical sequences are thus in one-to-one 
correspondence with permutations that can be produced with two parallel stacks.

In order to proceed further, we present three equivalent descriptions of what an operation sequence is. Recall what the basic scenario is: input items numbered consecutively from 1 through $n$ are processed by two stacks, each of which is capable of containing an arbitrarily large amount of data, but whose operations are limited to input $(I)$ and output $(O)$; an output operation produces the most recently entered item (i.e. items are processed in a last-in first-out fashion). Items are output as a sequence, and after all the input has been processed and the stacks emptied, the result is a permutation of the original input.

Operation sequences are encoded as words over the alphabet $\left\{I_{1}, I_{2}, O_{1}, O_{2}\right\}$, the subscripts determining which stack is referred to. Note that one cannot output from an empty stack, and that both stacks must be empty at the end. This means that a word over $\left\{I_{1}, I_{2}, O_{1}, O_{2}\right\}$ is an operation sequence if and only if it contains the same number of $I_{i}$ as $O_{i}$ letters for $i=1,2$, and, in each prefix, the number of $I_{i}$ letters is at least as great as the number of $O_{i}$ letters for $i=1,2$.

Let us call quarter plane walk a square lattice walk which begins at $(0,0)$, uses steps $E=(1,0)$, $N=(1,0), W=(-1,0)$ and $S=(0,-1)$ and remains in the quadrant $\{(x, y): x \geq 0, y \geq 0\}$. This walk is a loop if it ends at $(0,0)$. There is an obvious one-to-one correspondence between operation sequences and quarter plane loops (replace $I_{1}$ by $E, I_{2}$ by $N, O_{1}$ by $W$ and $O_{2}$ by $S$ ). Under this correspondence, the $(x, y)$ coordinate reached after processing a prefix of an operation sequence simply records the number of items in each stack at that point. The number of quarter plane loops consisting of $2 n$ steps is well known to be $C_{n} C_{n+1}$, where $C_{n}=\left(\begin{array}{c}2 n \\ n\end{array}\right) /(n+1)$ is the $n$th Catalan number [10, 1] .

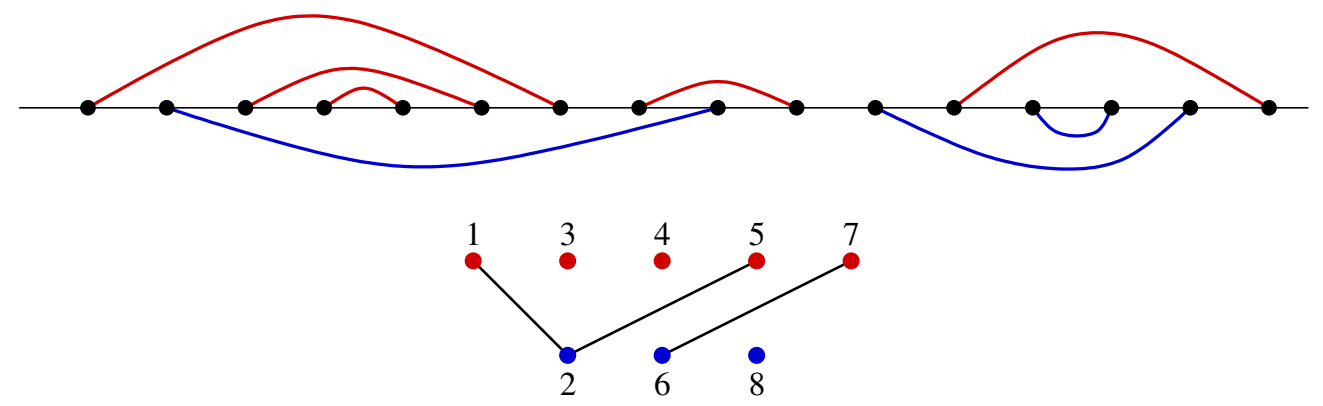

Fig. 3: The arch system associated with the operation sequence $I_{1} I_{2} I_{1} I_{1} O_{1} O_{1} O_{1} I_{1} O_{2} O_{1} I_{2} I_{1} I_{2} O_{2} O_{2} O_{1}$, and the associated graph. The arches are labelled using the left-to-right order of their left endpoint. This arch system has five connected components, and one left-right pair (between arches 2 and 5). The output permutation is 43125867.

A third perspective on these objects arises from considering them as bi-coloured arch systems (Figure 3. This is the two-dimensional counterpart of the standard bijection between Dyck paths and (onecoloured) arch systems [19, Exercise 6.19o]. For an operation sequence of length $2 n$, take $2 n$ points arranged along a line, labelled from 1 to $2 n$. These points represent time, that is, the $2 n$ steps of the operation sequence. For each item $k$ in $\{1, \ldots, n\}$, build an arch joining $i$ to $j$ where $i$ (resp. $j$ ) is the time at which $k$ is put in (resp. out of) a stack. If $k$ is processed by the first stack, the arch will be above the line (and will be thought of as red), and otherwise below the line (and thought of as blue). Observe that the set of arches above the line may not contain any crossings, nor can the one below the line - but there are no further restrictions on such systems. The operation sequence is easily recovered by scanning from left 
to right the $2 n$ points of the arch system, writing $I$ (resp. $O$ ) if an arch opens (resp. closes) at this point, and 1 (resp. 2) if this arch is above (resp. below) the line. Upon closing the supporting line into a cycle, an arch system can also be seen as a rooted planar cubic map with a distinguished Hamiltonian cycle. In this disguise, they were already considered by Tutte [21].

Let us observe that two sequences obtained from one another by commuting pairs of adjacent letters $I_{1} O_{2}$ or $I_{2} O_{1}$ are equivalent. An operation sequence outputs eagerly if it contains neither $I_{1} O_{2}$ nor $I_{2} O_{1}$ as a factor. In other words, if the next item of the permutation which it is producing is already present in one of the two stacks (necessarily at the top of the stack), then it is output immediately, before any other input (necessarily to the other stack) is carried out. Such sequences correspond to walks in the plane containing no $E S$ or $N W$ factor and to arch systems in which the left endpoint of an arch of one colour is never followed immediately by the right endpoint of an arch of the opposite colour - a configuration that we call a left-right pair (see Figure 3).

Lemma 1 (Pratt [18]) If a permutation can be produced by some operation sequence, then it can be produced by one that outputs eagerly.

A second source of ambiguity in operation sequences is the possibility of reflecting one or several (well chosen) arches in the horizontal line. We say that two arches of different colours cross if they cross once the one below the line is reflected. We sometimes consider the arches as vertices of a graph, two arches being adjacent if they cross (Figure 3 bottom). This graph is then bipartite. We refer to its connected components as the (connected) components of the arch system, and call a non-empty arch system connected if its corresponding graph is. In terms of operation sequences, or equivalently quarter plane loops, this means that no proper factor is an operation sequence.

Definition 2 An arch system is standard if the first arch of each component is red (that is, above the line). It is canonical if, in addition, it outputs eagerly.

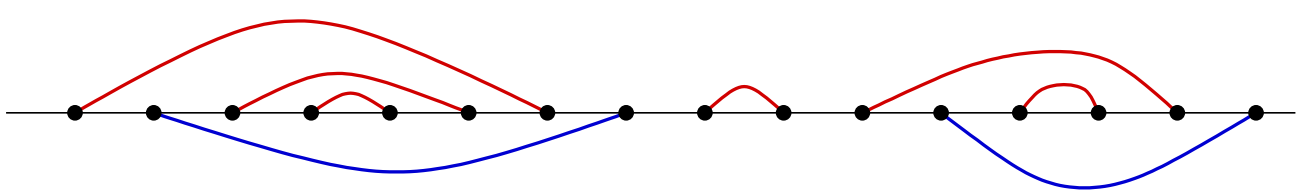

Fig. 4: The canonical arch system that is equivalent to the arch system of Figure 3 Note that the left-right pair created by edges 2 and 5 in Figure 3 has disappeared (in fact, these edges do not cross any more). Also, the colours of the two rightmost components (edges $6,7,8$ ) have been changed. The output permutation is still 43125867.

The following lemma is illustrated by Figure 4

Lemma 3 If a permutation can be produced by some operation sequence, then it can be produced by a canonical one.

A detailed but routine analysis yields:

Proposition 4 Every achievable permutation is produced by a unique canonical operation sequence.

Note that the concatenation of two canonical arch systems is a canonical arch system. We say that a non-empty canonical arch system is primitive if it cannot be written as a non-trivial concatenation. The corresponding permutations are also called primitive. 


\section{Exact enumeration}

In this section, we establish a system of functional equations that characterises the length generating function $S(t)$ of permutations that can be produced (or sorted) by two stacks in parallel:

$$
S(t)=1+t+2 t^{2}+6 t^{3}+23 t^{4}+103 t^{5}+513 t^{6}+2760 t^{7}+15741 t^{8}+O\left(t^{9}\right) .
$$

The first equation in this system characterises the generating function $\mathcal{Q}(a, u ; x, y)$ of quarter plane walks, counted by the length (variable $u$ ), the number of NW or ES corners (variable $a$ ), and the coordinates of their endpoint (variables $x$ and $y$ ). By setting $x=y=0$, and replacing $u$ by $\sqrt{u}$, one obtains the generating function $Q(a, u)$ of quarter plane loops counted by half length $(u)$ and NW or ES corners $(a)$ :

$$
Q(a, u)=1+2 u+(8+2 a) u^{2}+\left(44+24 a+2 a^{2}\right) u^{3}+O\left(u^{4}\right) .
$$

Equivalently, $Q(a, u)$ counts arch systems by the number of arches $(u)$ and the number of left-right pairs $(a)$. The last series involved in our system of equations is the generating function of connected standard arch systems, counted by the number of arches $(v)$ and the number of left-right pairs $(b)$ :

$$
C(b, v)=v+b v^{2}+b(b+2) v^{3}+O\left(v^{4}\right) .
$$

We find it convenient to use three different length variables $(t, u$ and $v$ ) and two different corner variables ( $a$ and $b$ ) when we perform substitutions in our generating functions.

Theorem 5 The generating function $\mathcal{Q}(a, u ; x, y) \equiv \mathcal{Q}(x, y)$ of quarter plane walks is characterised by:

$$
\begin{aligned}
& \left(1-u(x+\bar{x}+y+\bar{y})-u^{2}(a-1)(x \bar{y}+y \bar{x})\right) \mathcal{Q}(x, y)= \\
& 1-u \bar{y}(1+u x(a-1)) \mathcal{Q}(x, 0)-u \bar{x}(1+u y(a-1)) \mathcal{Q}(0, y),
\end{aligned}
$$

where $\bar{x}=1 / x$ and $\bar{y}=1 / y$. The generating function for quarter plane loops is thus

$$
Q(a, u)=\mathcal{Q}(a, \sqrt{u} ; 0,0) .
$$

The generating function $C(b, v)$ for connected standard arch systems is characterised by

$$
Q(a, u)=1+2 C\left(1-\frac{1-a}{Q}, u Q^{2}\right)
$$

where $Q$ stands for $Q(a, u)$. Finally, the generating function $S(t) \equiv S$ of permutations that can be produced by two stacks operating in parallel is characterised by

$$
S(t)=1+C\left(1-\frac{1}{S}, t S^{2}\right) .
$$

The equation defining $\mathcal{Q}(x, y)$ translates a simple recursive description of quarter plane walks, constructed by adding one or two steps at a time (see [7] for such arguments in the case of unweighted walks). The argument required to prove the other two results uses the structural decomposition of arch systems suggested by Figure 5

By relating the functional equation (2) to a compositional inversion of bivariate series, we can eliminate the series $C(b, v)$ from the system of Theorem 5 , and thus obtain an equation defining $S(t)$ in terms of $Q(a, u)$. This relation looks nicer when we introduce the generating function $S^{\bullet}(t)$ that counts primitive canonical operation sequences, defined at the end of Section 2 


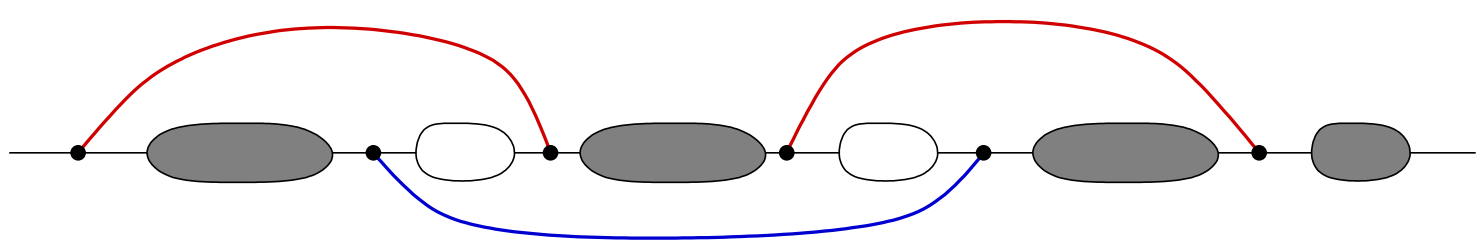

Fig. 5: The structure of an arch system: a connected system $c$ with $n$ arches (here, $n=3$ ), in which $2 n$ arbitrary arch systems are inserted. Here, $c$ has two left-right pairs. The arch systems that are inserted there (shown in white) destroy these left-right pairs, unless they are empty.

Corollary 6 The series $Q(a, u), S(t) \equiv S$ and $S^{\bullet}(t) \equiv S^{\bullet}$ that count quarter plane loops, sortable permutations and primitive sortable permutations respectively, are related by

$$
S=\frac{1}{1-S^{\bullet}} \quad \text { and } \quad Q\left(-S^{\bullet}, \frac{t}{\left(1+S^{\bullet}\right)^{2}}\right)=\frac{1+S^{\bullet}}{1-S^{\bullet}}
$$

\section{Corners in square lattice walks}

Our analysis of the asymptotic behaviour of the number of sortable permutations of length $n$, performed in the next section, relies on three conjectures, which have their own combinatorial interest.

Conjecture 7 The series $Q(a, u)$ is $(a+1)$-positive: i.e. there exists polynomials $P_{n}(b) \in \mathbb{N}[b]$ such that

$$
Q(a, u)=\sum_{n \geq 0} u^{n} P_{n}(a+1) .
$$

Of course, $Q(a, u)$ is a power series in $u$ with coefficients in $\mathbb{N}[a]$, and hence in $\mathbb{Z}[a+1]$. What is not clear is why the coefficient of $(a+1)^{k}$ should be non-negative. This has been checked on a computer up to half length $n=100$, using the functional equation (11). We conjecture this holds as well if we include a variable $s$ counting East steps.

Conjecture 8 For $a \geq-1$, the radius of convergence of $Q(a, \cdot)$ is

$$
\rho_{Q}(a)= \begin{cases}\frac{1}{(2+\sqrt{2+2 a})^{2}} & \text { if } a \geq-1 / 2, \\ -\frac{a}{2(1-a)^{2}} & \text { if } a \in[-1,-1 / 2] .\end{cases}
$$

We shall only use the above conjecture for $a \geq-1 / 2$.

Conjecture 9 The series $Q_{2}^{\prime}(a, u):=\frac{\partial Q}{\partial u}(a, u)$ is convergent at $u=\rho_{Q}(a)$ for $a \geq-1 / 3$.

In the following subsections, we gather some evidence for these conjectures. In particular, we prove Conjectures 7 and 8 for general loops and for loops confined to the upper half plane. (Conjecture 9 does not hold for these more general loops.) The fact that Conjecture 8 holds for general loops and half plane loops is reminiscent of a recent result according to which the growth constant of (unweighted) loops confined to a wedge is independent of this wedge [8, Sec. 1.5]. 


\subsection{Some results on quarter plane loops}

Proposition 10 The series $Q(a, s, u)$ counting quarter plane loops by the half length (u), the number of East steps ( $s$ ), and the number of $N W$ and ES factors (a) satisfies

$$
Q(1, s, u)=\sum_{i, j \geq 0}\left(\begin{array}{c}
2 i+2 j \\
2 i
\end{array}\right) C_{i} C_{j} s^{i} u^{i+j}
$$

where $C_{i}=\left(\begin{array}{c}2 i \\ i\end{array}\right) /(i+1)$ is the ith Catalan number. The value at $a=-1$ is just as remarkable:

$$
Q(-1, s, u)=\sum_{i, j \geq 0}\left(\begin{array}{c}
i+j \\
i
\end{array}\right) C_{i} C_{j} s^{i} u^{i+j}
$$

The results dealing with $Q(1, s, t)$ are well-known and easy to prove [10]. For the case $a=-1$, we work from the functional equation (1), refined with a variable $s$. The proof is inspired by recent progress on quarter plane walks with general steps [7]. It still lacks a combinatorial explanation.

Let us now discuss the radius of convergence of $Q(a, \cdot)$.

Proposition 11 For a fixed, let $\rho_{Q}(a)$ be the radius of convergence of $Q(a, \cdot)$. Then

$$
\rho_{Q}(-1)=\frac{1}{8}, \quad \rho_{Q}(1)=\frac{1}{16},
$$

and $\rho$ is non-increasing function on $[0,+\infty)$, continuous on $(0,+\infty)$. Moreover, for $a \geq 0$,

$$
\rho_{Q}(a) \geq \frac{1}{(2+\sqrt{2+2 a})^{2}} .
$$

The series $Q_{2}^{\prime}\left(a, \rho_{Q}(a)\right)$ converges for $a=-1$ and $a=1$.

If $Q(a, u)$ is $(a+1)$-positive, then $\rho_{Q}$ is non-increasing on $[-1,+\infty)$ and continuous on $(-1,+\infty)$.

The values at $a=1$ and $a=-1$ follow from Proposition 10 The lower bound is obtained by counting all walks by length and corners. The continuity result is standard for series with coefficients in $\mathbb{N}[a]$ and affine degree.

\subsection{Unconfined loops}

We now consider unconfined loops. Their generating function can be obtained by two successive coefficient extractions in the rational generating function that counts all walks and keeps track of their final position. This allows us to prove that Conjectures $7((a+1)$-positivity) and 8 (radius of convergence) hold for these loops.

Proposition 12 The generating function $\mathcal{W}(a, s, t ; x, y)$ counting square lattice walks by the number of horizontal steps $(s)$, the number of vertical steps $(t)$, the number of ES and NW corners (a) and the coordinates of the endpoint $(x, y)$ is rational, and given by

$$
\mathcal{W}(a, s, t ; x, y)=\frac{1}{1-s(x+\bar{x})-t(y+\bar{y})-s t(a-1)(x \bar{y}+\bar{x} y)} .
$$


The generating function that only counts loops is D-finite, and given by

$$
\mathcal{W}_{0,0}(a, s, t):=\left[x^{0} y^{0}\right] \mathcal{W}(a, s, t ; x, y)=\sum_{j \geq 0}\left(\begin{array}{c}
2 j \\
j
\end{array}\right) t^{2 j} \mathcal{W}_{j}(a, s)
$$

where

$$
\mathcal{W}_{j}(a, s)=\left[x^{0}\right] \frac{(1-s x(1-a))^{j}(1-s \bar{x}(1-a))^{j}}{(1-s(x+\bar{x}))^{2 j+1}} .
$$

The generating function $A(a, s, t):=\sum_{j \geq 0} t^{2 j} \mathcal{W}_{j}(a, s)$ is biquadratic, and can be written as

$$
A(a, s, t)=\frac{1+t^{2}\left(1-a^{2}\right) T}{\left(1-t^{2}\left(a^{2}-1\right) T\right)^{2}-t^{2}(1+2(a+1) T)^{2}} \sqrt{\frac{1+4 T-t^{2}(a-1)^{2} T}{1-t^{2}(a-1)^{2} T}},
$$

where $T \equiv T(a, s, t)$ is the unique series in $\mathbb{Q}[a][[s, t]]$ that satisfies $T(a, 0, t)=0$ and

$$
T=s^{2} \frac{1+4 T-(a-1)^{2} t^{2} T}{1-t^{2}-t^{2}(a+1)^{2} T} .
$$

The expression of $\mathcal{W}(a, s, t ; x, y)$ follows from the same recursive construction of walks as the functional equation (1) for quarter plane walks. Extracting the coefficient of $x^{0} y^{0}$ gives (5). If we drop the coefficient $\left(\begin{array}{c}2 j \\ j\end{array}\right)$, this becomes the constant term (in $x$ ) of a rational series, and thus an algebraic series $A(a, s, t)$. We perform the constant term extraction using a partial fraction expansion in $x$, as described for instance in [19, Thm. 6.3.3]. This gives a biquadratic equation for $A$, which we parametrise using (8).

Proposition 13 The series $\mathcal{W}_{0,0}(a, s, t)$ that counts unconfined loops is $(a+1)$-positive. Moreover,

$$
\mathcal{W}_{0,0}(1, s, t)=\sum_{i, j \geq 0} s^{2 i} t^{2 j}\left(\begin{array}{c}
2 i+2 j \\
2 i
\end{array}\right)\left(\begin{array}{c}
2 i \\
i
\end{array}\right)\left(\begin{array}{c}
2 j \\
j
\end{array}\right)
$$

while

$$
\mathcal{W}_{0,0}(-1, s, t)=\sum_{i, j \geq 0} s^{2 i} t^{2 j}\left(\begin{array}{c}
i+j \\
i
\end{array}\right)\left(\begin{array}{c}
2 i \\
i
\end{array}\right)\left(\begin{array}{c}
2 j \\
j
\end{array}\right) .
$$

We first establish $(a+1)$-positivity for $T$ using $(8)$, then for $A$ using (7). This is equivalent to saying that $\mathcal{W}_{0,0}(a, s, t)$ is $(a+1)$-positive. The expression of $\mathcal{W}_{0,0}(1, s, t)$ is combinatorially clear, while the expression of $\mathcal{W}(-1, s, t)$ follows by specializing Proposition 12 to $a=-1$.

Proposition 14 Let $a \geq-1$. The series $\mathcal{W}_{0,0}(a, \sqrt{u}, \sqrt{u})$ that counts unconfined loops walks by half length and corners has radius of convergence given by (3).

We first compute a differential equation, of order 2 , satisfied by this D-finite series. The radius is found among the roots of the coefficient of the second derivative. We select the correct root, depending on the value of $a$, by combining $(a+1)$-positivity, a continuity argument, and the known values $1 / 16$ and $1 / 8$ at $a=1$ and $a=-1$, respectively. 


\subsection{Half plane walks}

We obtain similar results for loops confined to the upper half plane $\{(x, y): y \geq 0\}$.

Proposition 15 The generating function of half plane loops, counted by horizontal steps $(s)$, vertical steps ( $t$ ), and NW and ES factors (a), is

$$
\mathcal{H}_{0,0}(a, s, t)=\sum_{j \geq 0} \frac{1}{j+1}\left(\begin{array}{c}
2 j \\
j
\end{array}\right) t^{2 j} \mathcal{W}_{j}(a, s),
$$

where $\mathcal{W}_{j}(a, s)$ is given by (6). It is $(a+1)$-positive. Moreover,

$$
\mathcal{H}_{0,0}(1, s, t)=\sum_{i, j \geq 0} s^{2 i} t^{2 j} \frac{1}{j+1}\left(\begin{array}{c}
2 i+2 j \\
2 i
\end{array}\right)\left(\begin{array}{c}
2 i \\
i
\end{array}\right)\left(\begin{array}{c}
2 j \\
j
\end{array}\right)
$$

while

$$
\mathcal{H}_{0,0}(-1, s, t)=\sum_{i, j \geq 0} s^{2 i} t^{2 j} \frac{1}{j+1}\left(\begin{array}{c}
i+j \\
i
\end{array}\right)\left(\begin{array}{c}
2 i \\
i
\end{array}\right)\left(\begin{array}{c}
2 j \\
j
\end{array}\right) .
$$

For $a \geq-1$, the series $\mathcal{H}_{0,0}(a, \sqrt{u}, \sqrt{u})$ that counts half space loops by half length and corners has radius of convergence given by [3].

These results follow from those obtained for unconfined walks, using the following proposition.

Proposition 16 Let $v$ be a word on $\{N, S\}$. The generating function of walks whose vertical projection is $v$, counted by the number of horizontal steps $(s)$, the abscissa of the endpoint $(x)$ and the number of $N W$ and SE factors (a), only depends on $|v|_{N}$ and $|v|_{S}$.

The proof is simple, once one realises that the number of NW and ES corners is distributed as the number of WN and SE corners, as stated in the following result (discovered independently by Olivier Bernardi and Julien Courtiel; personal communications).

Lemma 17 There exists an involution on square lattice paths that exchanges the number of $N W$ and $W N$ factors, fixes the number of ES or SE factors, and leaves the horizontal and vertical projections unchanged.

Consequently, for any pair of walks $v \in\{N, S\}$ and $w \in\{E, W\}$, the following bi-statistics of corners are equidistributed in the set of walks that project vertically on $v$ and horizontally on $w:$ (NW,ES), $(W N, E S),(W N, S E)$ and $(N W, S E)$.

\section{Asymptotic Analysis}

Recall the relationship between the series $Q(a, u)$ and $S \equiv S(t)$ established in Corollary 6

$$
Q\left(-S^{\bullet}, \frac{t}{\left(1+S^{\bullet}\right)^{2}}\right)=\frac{1+S^{\bullet}}{1-S^{\bullet}}
$$

with $S=1 /\left(1-S^{\bullet}\right)$. Our main theorem tells us that $S(t)$ becomes singular when the pair $\left(-S^{\bullet}, t(1+\right.$ $\left.S^{\bullet}\right)^{-2}$ ) reaches the critical curve of $Q$, namely $\left\{\left(a, \rho_{Q}(a)\right),-1 \leq a\right\}$ where $\rho_{Q}(a)$ is the radius of the series $Q(a, \cdot)$. However, this theorem relies on the conjectures studied in the previous section. 
Theorem 18 Assume that the series $Q(a, u)$ is $(a+1)$-positive, and that $Q_{2}^{\prime}\left(a, \rho_{Q}(a)\right)<\infty$ for $-1 / 3 \leq$ $a \leq 0$. Let $t_{c}$ be the radius of convergence of $S=1 /\left(1-S^{\bullet}\right)$. Then $t /\left(1+S^{\bullet}(t)\right)^{2}$ increases on the interval $\left[0, t_{c}\right]$, and on this interval,

$$
\frac{t}{\left(1+S^{\bullet}(t)\right)^{2}} \leq \rho_{Q}\left(-S^{\bullet}(t)\right),
$$

with equality if and only if $t=t_{c}$. Moreover, $S^{\bullet}\left(t_{c}\right) \leq 1 / 3$.

We can make the value of $t_{c}$ more explicit thanks to the conjectured expression of $\rho_{Q}(a)$ (Conjecture 8 .

Corollary 19 Assume that the assumptions of the above theorem hold, as well as (the first part of) Conjecture 8 . Then the radius of convergence of $S$ is

$$
t_{c}=\left(1-\frac{\sqrt{2+2 a}}{2}\right)^{2}
$$

where $a=-S^{\bullet}\left(t_{c}\right)$ satisfies

$$
Q\left(a, \frac{1}{(2+\sqrt{2+2 a})^{2}}\right)=\frac{1-a}{1+a} .
$$

Numerically, $a \simeq-0.1477$ and

$$
1 / t_{c}=\lim \sup s_{n}^{1 / n} \simeq 8.29 .
$$

The proof of these results is rather delicate, first because we have to deal with bivariate series, and then because $-S^{\bullet}$ is negative. The latter difficulty is partially alleviated by the (conjectured) $(a+1)$-positivity of $Q$. The main ingredients of the proof are the implicit function theorem, Pringsheim's theorem, and the open mapping theorem in two variables.

Acknowledgements. We are indebted to Olivier Bernardi, Julien Courtiel, Kilian Raschel, Alin Bostan, Cyril Banderier, Pierre Lairez for helpful and interesting discussions and thank LaBRI for its hospitality to the first author in visits in 2008 and 2012.

\section{References}

[1] Bernardi, O. Bijective counting of tree-rooted maps and shuffles of parenthesis systems. Electron. J. Combin. 14, 1 (2007), Research Paper 9, 36 pp. (electronic).

[2] BónA, M. A survey of stack-sorting disciplines. Electron. J. Combin. 9,2 (2002/03), Article 1.

[3] Bóna, M. Combinatorics of Permutations, Second Edition. Discrete Mathematics and Its Applications. Taylor \& Francis, 2012.

[4] Bostan, A., And Kauers, M. The complete generating function for Gessel walks is algebraic. Proc. Amer. Math. Soc. 138, 9 (2010), 3063-3078. With an appendix by Mark van Hoeij.

[5] Bostan, A., Raschel, K., And Salvy, B. Non-D-finite excursions in the quarter plane. $J$. Combin. Theory Ser. A 121 (2014), 45-63. 
[6] Bousquet-Mélou, M. Walks in the quarter plane: Kreweras' algebraic model. Ann. Appl. Probab. 15, 2 (2005), 1451-1491.

[7] Bousquet-Mélou, M., And Mishna, M. Walks with small steps in the quarter plane. Contemp. Math. 520 (2010), 1-40.

[8] Denis Denisov, V. W. Random walks in cones. Ann. Probab. (to appear). arXiv:1110.1254.

[9] Even, S., And ITAI, A. Queues, stacks, and graphs. In Theory of machines and computations (Proc. Internat. Sympos., Technion, Haifa, 1971). Academic Press, New York, 1971, pp. 71-86.

[10] Guy, R. K., Krattenthaler, C., And Sagan, B. E. Lattice paths, reflections, \& dimensionchanging bijections. Ars Combin. 34 (1992), 3-15.

[11] Kauers, M., Koutschan, C., And Zeilberger, D. Proof of Ira Gessel's lattice path conjecture. Proc. Natl. Acad. Sci. USA 106, 28 (2009), 11502-11505.

[12] Kitaev, S. Patterns in Permutations and Words. Monographs in theoretical computer science. Springer, 2011.

[13] KnUth, D. E. The art of computer programming. Vol. 1: Fundamental algorithms. AddisonWesley Series in Computer Science and Information Processing. London, 1968.

[14] KURKova, I., AND RASChEl, K. On the functions counting walks with small steps in the quarter plane. Publ. Math. Inst. Hautes Études Sci. 116 (2012), 69-114.

[15] Linton, S., RušKuc, N., And Vatter, V., Eds. Permutation patterns, vol. 376 of London Mathematical Society Lecture Note Series. Cambridge University Press, Cambridge, 2010. Papers from the 5th Conference held at the University of St. Andrews, St. Andrews, June 11-15, 2007.

[16] Murphy, M. M. Restricted permutations, antichains, atomic classes and stack sorting. PhD thesis, University of St. Andrews, 2002.

[17] Pierrot, A., And Rossin, D. 2-Stack sorting is polynomial. ArXiv:1304.2860, 2013.

[18] Pratt, V. R. Computing permutations with double-ended queues, parallel stacks and parallel queues. In STOC '73: Proceedings of the fifth annual ACM symposium on Theory of computing (New York, NY, USA, 1973), ACM Press, pp. 268-277.

[19] Stanley, R. P. Enumerative combinatorics. Vol. 2, vol. 62 of Cambridge Studies in Advanced Mathematics. Cambridge University Press, Cambridge, 1999. With a foreword by Gian-Carlo Rota and appendix 1 by Sergey Fomin.

[20] Tarjan, R. Sorting using networks of queues and stacks. J. Assoc. Comput. Mach. 19 (1972), 341-346.

[21] Tutte, W. T. A census of Hamiltonian polygons. Canad. J. Math. 14 (1962), 402-417.

[22] UngER, W. The complexity of colouring circle graphs (extended abstract). In STACS (1992), A. Finkel and M. Jantzen, Eds., vol. 577 of Lecture Notes in Computer Science, pp. 389-400. 